\title{
Im Namen der Gerechtigkeit
}

\author{
von Jasmin Häcker, Bernd Raffelhüschen und Jörg Schoder
}

Der Begriff der Gerechtigkeit ist zwar vieldiskutiert, aber nicht eindeutig operationalisiert. Mit Blick auf seine Rolle im politischen Prozess besteht vor diesem Hintergrund die Gefahr, dass das öffentliche Gerechtigkeitsempfinden für die Durchsetzung von Partikularinteressen missbraucht wird. Ein Beitrag zur Lösung dieser grundlegenden Problematik des politischen Prozesses kann in der Erhöhung der Transparenz liegen. Im Sinne von Kelsen geht es also darum, ,, bessere Fragen“ zu stellen. Politische Maßnahmen wirken sich meist auf mehrere Dimensionen eines umfassend definierten Gerechtigkeitsbegriffs aus. Die Methodik der Generationenbilanzierung kann dabei hilfreich sein, mögliche Konflikte zwischen intra- und intergenerativer Gerechtigkeit zu identifizieren, ohne jedoch den Anspruch zu erheben, Gerechtigkeit messen zu können.

The debate on issues of justice is as old as mankind; however, no unambiguous operationalisation has been agreed upon so far. Regarding its role in practical politics, the term bears the risk of interest groups abusing the common perception of justice for their objectives. Improved transparency in political decision-making can help to avoid these dangers. According to Kelsen, it is all about asking ,, better questions“. The term justice has often several dimensions, which are affected differently, depending on the underlying policy. The method of generational accounting can be a useful tool for identifying conflicts between inter-and intragenerational justice, without claiming to be able to measure justice altogether.

„Was ist Gerechtigkeit? Keine andere Frage ist so leidenschaftlich erörtert, für keine andere Frage so viel kostbares Blut, so viel bittere Tränen vergossen worden, über keine andere Frage haben die erlauchtesten Geister - von Platon bis Kant - so tief gegrübelt. Und doch ist diese Frage heute so unbeantwortet wie je. Vielleicht, weil es eine jener Fragen ist, für die die resignierte Weisheit gilt, daß der Mensch nie eine endgültige Antwort findet, sondern nur suchen kann, besser zu fragen.“

Hans Kelsen, Was ist Gerechtigkeit?, Stuttgart, 2000, 9.

\section{Einleitung}

Das Vertrauen der Deutschen in die Marktwirtschaft schwindet. Waren nach einer Umfrage des Instituts für praxisorientierte Sozialforschung im Jahr 2000 
noch $70 \%$ davon überzeugt, dass sich die Soziale Marktwirtschaft bewährt hat, so sind es heute nur noch $52 \%$. Als Erklärung für diesen Vertrauensverlust wird häufig ein Gerechtigkeitsdefizit gesehen, dem aufgrund des internationalen Wettbewerbsdrucks in den letzten Jahren durch sozialpolitische Maßnahmen nicht ausreichend entgegengewirkt werden konnte. Als Beleg für diesen Zusammenhang wird gerne der jüngste Armuts- und Reichtumsbericht der Bundesregierung herangezogen, der ein bedenkliches Bild zeichnet und so in der Bevölkerung Befürchtungen eines drohenden materiellen und sozialen Abstiegs fördert.

In der politischen Diskussion und in den Medien wird mit diesen Ängsten zudem oft leichtfertig gespielt. Offensichtliche Missstände werden an Einzelbeispielen dargestellt und unzulässig verallgemeinert, mit dem Tenor, dass dies in einem so reichen Land wie Deutschland nicht vorkommen dürfe. Auf Lösungsvorschläge und Lösungsansätze wird jedoch meist verzichtet, wohl wissend, dass diese in den meisten Fällen eher unpopulär oder aber utopisch wären. Gerade im Hinblick auf soziale Fragen entsteht zunehmend der Eindruck einer Tabuisierung unbequemer Botschaften. Die Forderung nach mehr Gerechtigkeit, ob als soziale Gerechtigkeit oder als Generationengerechtigkeit, wird dabei nicht selten zum politischen Totschlagargument. Denn wer will schon als ungerecht oder nicht sozial bzw. unsozial gelten?

Dieser Beitrag verfolgt das Ziel, einen Beitrag zur Versachlichung der Diskussion über die zentralen Zukunftsfelder der deutschen Sozialpolitik zu leisten und dabei auf gängige und bedeutsame Mechanismen und Wechselbeziehungen im politischen Entscheidungsprozess einzugehen und mögliche Konsequenzen herauszuarbeiten. Eine besondere Rolle spielt dabei das Verhältnis von Medien, Politik und Wissenschaft. Soziale Gerechtigkeit und Generationengerechtigkeit werden in diesem Zusammenhang oft inflationär und unreflektiert verwendet. Zur Klärung werden deshalb beide Begriffe im zweiten Abschnitt zunächst inhaltlich abgegrenzt und theoretisch analysiert. In einem dritten Abschnitt wird dann das für die praktische Politik entscheidende Problem ihrer Operationalisierung thematisiert und am Beispiel der aktuellen Armutsdebatte kurz anschaulich diskutiert. Dabei wird gezeigt, dass veröffentlichte Zahlen ohne genaue Kenntnis der theoretischen und methodischen Hintergründe schnell $\mathrm{zu}$ einer falschen Wahrnehmung in der Bevölkerung führen können. Dies wiederum kann zu einem akuten politischen Handlungsdruck führen, durch den langfristig orientierte politische Strategien erschwert oder verhindert werden. Danach wird in Abschnitt vier am Beispiel der Einführung der Sozialen Pflegeversicherung sowie ihrer jüngsten Reform gezeigt, dass im Namen der Gerechtigkeit manchmal 
Maßnahmen gefordert und verabschiedet werden, die nicht selten dem naiven Gerechtigkeitsempfinden sogar widersprechen müssten.

\section{Gerechtigkeit - Ideal oder Worthülse?}

Fragen der Gerechtigkeit sind so alt wie die Geschichte des menschlichen $\mathrm{Zu}$ sammenlebens. Seit Aristoteles existiert die Unterscheidung in Verhaltens- und Verteilungsgerechtigkeit. Heute ist Verhaltensgerechtigkeit nach Streit dann gegeben, wenn eine Gleichbehandlung vor dem Recht ${ }^{1}$ bzw. Diskriminierungsfreiheit vorliegt, die er als gleiche formale Freiheit bezeichnet. ${ }^{2}$ Gleiche formale Freiheit sichert demzufolge Handlungsfreiheit und bedeutet für den wirtschaftlichen Bereich zwangsläufig die Notwendigkeit, Kooperationen bzw. Kompromisse auf freiwilliger, eigenverantwortlicher Basis zu schließen. Dem prozessorientierten Begriff der formalen Gerechtigkeit steht der vornehmlich ergebnisorientierte Begriff der Verteilungsgerechtigkeit gegenüber, von Streit auch als materiale Gleichheit bezeichnet.

In der Ökonomie wurden Fragen der Gerechtigkeit vor allem in der Zeit der Klassiker thematisiert. So stellt beispielsweise John Stuart Mill fest:

„Ich weiß nicht, weshalb man sich dazu beglückwünschen soll, dass Menschen, die bereits reicher sind als irgendeiner nötig hat, ihre Mittel verdoppeln, um etwas zu verbrauchen, was außer der Schaustellung ihres Reichtums nur wenig oder gar keine Freuden verschafft.“3

Mill stand wie Bentham in der Tradition des klassischen Utilitarismus, der das größte Glück der größten Zahl als gesellschaftlichen Idealzustand definierte. Konzeptionell führt dieser Ansatz aber zum Problem der fehlenden intersubjektiven Vergleichbarkeit - denn Nutzenempfindungen sind individuell bestimmt und deshalb recht unterschiedlich. Mit der paretianischen Wohlfahrtsökonomik gewann die bereits bei Adam Smith angelegte Trennung von Allokation und Distribution zunehmend an Gewicht. Dadurch wurden Verteilungsfragen zu Randbereichen der Wirtschaftswissenschaften. Die Allokation, und damit die Frage, wie viele Güter überhaupt verteilt werden können, wurde zur ersten Zuständigkeit der Disziplin erklärt. Mit seiner Theorie der Verfahrensgerechtigkeit überwindet

1 Recht ist dabei durch die jeweils gegebenen Rechtsnormen und Gesetze definiert.

2 Streit, M.: Freiheit und Gerechtigkeit. Ordnungspolitische Aspekte zweier gesellschaftlicher Grundwerte, in: Ordo, Jahrbuch für die Ordnung von Wirtschaft und Gesellschaft, 39 (1988), 33-53.

3 Mill, J.S.: Über den stationären Zustand (1848/1921), in: Siebert, H. (Hg.): Umwelt und wirtschaftliche Entwicklung. Darmstadt, 1979, 15-22, hier 19. 
Rawls in der Folge die Probleme einer ergebnisorientierten Gerechtigkeitsauffassung. Ihm geht es dabei um die Gerechtigkeit als fairen Prozess - nicht im Ergebnis ex post, sondern als Chancengleichheit ex ante, die unter dem Schleier der Unwissenheit Ungleichheit nur zulässt, wenn der am schlechtesten Gestellte etwas davon hat.

Libertäre Ökonomen in der Tradition Hayeks vertreten ebenfalls einen verfahrensorientierten Gerechtigkeitsbegriff. Das gesellschaftliche Koordinationsproblem wird durch eigenverantwortliches Handeln auf der Grundlage allgemeingültiger Regeln gelöst. Die Wahrnehmung der für alle gleichen Rechte muss jedoch nicht zu gleichen „oder auch nur ähnlichen ökonomischen Ergebnissen und sozioökonomischen Positionen" führen. ${ }^{4}$ Lediglich die Verhaltensweise, nicht aber die Ergebnisse des so betrachtet „fairen“ Spiels können als gerecht oder ungerecht bewertet werden. Letztere sind allenfalls als rechtmäßig anzusehen. Die Verfahrensorientierung schließt allerdings nicht aus, dass sich die Gesellschaftsmitglieder hinter dem Schleier der Unwissenheit für eine Mindestsicherung entscheiden. An diesen Ansätzen bzw. dieser Betrachtungsweise kritisiert Sen, dass die unbedingte Geltung bestimmter Rechte durchaus ungeeignete Ergebnisse hervorbringen kann. Er sieht es als ein Verdienst der Wohlfahrtsökonomik, dass die Ergebnisse stärker ethisch berücksichtigt wurden, und versucht mit seinem Ansatz eine Verknüpfung der beiden Pole von Ergebnis- und Verfahrensgerechtigkeit. ${ }^{5}$

Streit erwähnt in einer Fußnote eine Repräsentativerhebung aus den USA, aus der hervorgeht, dass Marktergebnisse durchaus als Folge gerechten Verhaltens akzeptiert werden:

„Wenn jedoch die gleichen Befragten mit empirischen Befunden über Verteilungen konfrontiert werden, neigen sie zu Korrekturen, ohne die auch für sie erkennbare Inkonsistenz auflösen zu können.“6

Der nach Konsistenz strebende (prozessorientierte) Theoretiker mag daran verzweifeln, die „empirische Gesellschaft“ benötigt jedoch Lösungen. Die Formu-

5 Sen, A.: Ökonomie für den Menschen. Wege zu Gerechtigkeit und Solidarität in der Marktwirtschaft, München u.a., 2000. Sen spricht sich für die Betrachtung des ,tatsächlich von den Menschen geführten Lebens" aus. Entscheidend sind nicht die Güter und auch nicht der Nutzen. Vielmehr geht es darum, welche Eigenschaften die Güter haben und was der Einzelne damit anfängt, d.h. welche tatsächlichen Möglichkeiten (functionings) existieren. Entscheidend hierfür sind dabei die Verwirklichungschancen (capabilities), die natürlich mit Freiheit eng verbunden sind.

6 Streit, M., a. a. O., 43. 
lierung der deutschen Verfassung trägt offensichtlich dem menschlichen Empfinden, das in der Befragung zum Ausdruck kommt, Rechnung und entspricht damit auch der Auffassung Sens, der einen Kompromiss aus Verfahrens- und Ergebnisorientierung einfordert. Artikel 20 GG bestimmt: „Die Bundesrepublik Deutschland ist ein demokratischer und sozialer Bundesstaat. " Der mit dieser Formulierung gegebene Interpretationsspielraum überlässt die konkrete Auslegung der praktischen Politik, die eine Gewichtung der grundlegenden und allgemein anerkannten konstituierenden Prinzipien des Sozialstaats - der Selbstverantwortlichkeit bzw. Subsidiarität und der Solidarität - vornehmen muss. Daraus ergibt sich auch, dass die Interpretation der Sozialstaatlichkeit immer auch vom Zeitgeist mitbestimmt wird.

Dieser Interpretationsspielraum wurde in der deutschen Nachkriegsgeschichte vor allem in Richtung der Sozialen Marktwirtschaft ausgelegt. Das von MüllerArmack und Erhard entwickelte wirtschaftspolitische Leitbild beruht auf den Forderungen der Freiburger Schule (Ordoliberalismus). Aufgabe des Staates ist es primär, eine funktionsfähige Wettbewerbsordnung zu gewährleisten, diese jedoch um sozialpolitische Ziele zu ergänzen und so die Positionen der (christlichen) Soziallehre und des Liberalismus zu verbinden. Auf diese Weise können die Vorteile der marktwirtschaftlichen Ordnung zur Maximierung des Wohlstands genutzt, aber auch eventuell auftretende soziale Spannungen und Gegensätze abgebaut bzw. beseitigt werden. Die Sozialpolitik erhält damit eine zentrale Bedeutung. Im weiteren Sinne gehören dazu sämtliche institutionalisierten Formen politischen Handelns, die im Hinblick auf soziale Sicherheit und soziale Gerechtigkeit durch den Staat bzw. Körperschaften des öffentlichen Rechts unternommen werden. ${ }^{7}$ Sozialpolitik dient mithin der Korrektur unerwünschter Ergebnisse des marktwirtschaftlich organisierten Koordinierungsprozesses. Sie definiert in diesem Kontext also einen ergebnisorientierten Gerechtigkeitsbegriff. Ein so verwendeter Begriff der sozialen Gerechtigkeit ist aber begriffslogisch durchaus problematisch:

7 Die mit dem von Weber eingeleiteten Werturteilsstreit aufgeworfene Frage, ob es sich um einen Begriff handelt, der rein im Zusammenhang mit der praktischen Politik steht, oder ob er der Wissenschaft zugänglich ist, konnte nicht abschließend geklärt werden, wenngleich die Sozialpolitik bis heute Gegenstand verschiedener Wissenschaftsdisziplinen ist (u. a. Volkswirtschaftslehre, Soziologie, Politikwissenschaft). 
„Wenn Gleichbehandlung [durch das Recht] zu ungleichen Ergebnissen führt, dann erfordert Angleichung, dass von einer Gleichbehandlung abgewichen werden muss. Angleichung macht Diskriminierung unumgänglich.“ ${ }^{8}$

Die Verlautbarungen des Club of Rome und die Wachstumsskepsis dieser Zeit wirkten sich auch auf den Zeitgeist aus. Zur sozialen Gerechtigkeit kamen nun noch die Begriffe der Generationengerechtigkeit und der Nachhaltigkeit. Der Begriff der Generationengerechtigkeit wird umgangssprachlich und allgemein im Zusammenhang mit der Verteilung der Möglichkeiten der Bedürfnisbefriedigung zwischen heutigen und künftigen Generationen verwendet. Präziser ist damit eine intergenerative Gerechtigkeit gemeint. In der Definition von Generation werden verschiedene Dimensionen bzw. Bezugsfelder unterschieden. So wird zwischen einem intratemporalen und einem intertemporalen Generationenbegriff differenziert.

Intratemporal gesehen leben stets mehrere Generationen gleichzeitig, wobei die Zuordnung i.d.R. von der biologischen Reproduktion bestimmt wird, so dass eine Generation ca. 30 Jahrgänge umfasst. ${ }^{9}$ Die intertemporale Sicht bezeichnet die zu einem Zeitpunkt auf der Erde lebenden Menschen als Generation. Entsprechend kann auch der Begriff der Generationengerechtigkeit in unterschiedlicher Weise inhaltlich beschrieben werden: als intergenerative Gerechtigkeit und als intragenerative Gerechtigkeit. Letztere wird in der Regel mit der bereits erwähnten Verteilungsgerechtigkeit bzw. der sozialen Gerechtigkeit unter den heute Lebenden gleichgesetzt, während erstere - wie bereits festgestellt - die landläufige Auffassung des Begriffs Generationengerechtigkeit widerspiegelt. Im Kontext der intergenerationalen Gerechtigkeit wird dabei oft der von der Brundt-

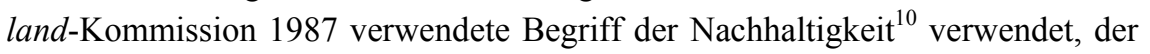
seinerzeit auf die ökologische Dimension zielte:

8 Streit, M., a. a. O., 43.

9 Genau genommen ist die statistische Abgrenzung einer Generation mit dem sog. Proliferationsalter gleichzusetzen, d.h. mit dem durchschnittlichen Alter der Mütter bei der Geburt ihres ersten lebendgeborenen Kindes. Dieses betrug in Deutschland nach Angaben des Statistischen Bundesamtes im Jahr 2005 genau 30,1 Jahre.

10 Der Begriff Nachhaltigkeit wird allerdings zu Unrecht der ökologischen Diskussion der letzten Jahrzehnte zugeordnet. Tatsächlich ist er viel älter und wurde bereits im Jahr 1713 durch Hans Carl von Carlowitz in der deutschen Forstwirtschaft geprägt, vgl. Grober, U.: Der Erfinder der Nachhaltigkeit, in: Die Zeit vom 25.11.1999, 98. Mit der fiskalischen Nachhaltigkeit befasste sich 1821 erstmals David Ricardo (Ricardo, D.: Über die Grundsätze der politischen Ökonomie und der Besteuerung, hg. v. H. Kurz und C. Gehrke, Marburg, 1994). 
„Dauerhafte Entwicklung ist Entwicklung, die die Bedürfnisse der Gegenwart befriedigt, ohne zu riskieren, dass künftige Generationen ihre eigenen Bedürfnisse nicht befriedigen können. “11

Generationengerechtigkeit wie auch soziale Gerechtigkeit beschreiben somit Dimensionen eines umfassenden Gerechtigkeitsbegriffs. Unklar bzw. offen bleibt, welche Dimension den Vorrang hat - denn nicht selten ergeben sich zwischen beiden Zielkonflikte. Ein solcher Zielkonflikt besteht gerade in den bedeutendsten Zweigen der Sozialpolitik - den umlagefinanzierten Sozialversicherungen. Beide Begriffe beschreiben verständliche Anliegen, ein theoretisch überlegener und akzeptabler Kompromiss ist wissenschaftlich jedoch nicht zu begründen. Vielmehr ist es die Aufgabe der Politik, einen entsprechenden Interessenausgleich zu schaffen.

\section{Gerechtigkeit in der praktischen Politik}

Die Diskussion des Gerechtigkeitsbegriffs hat gezeigt, dass Gerechtigkeit etwas mit Gleichheit zu tun hat. Dabei ist die Frage nach Dimension, Ausmaß und Niveau der Gleichheit in der Realität schwierig und theoretisch nicht eindeutig zu beantworten. Angesichts dieses inhaltlichen Problems werden die Begriffe der sozialen Gerechtigkeit und der Generationengerechtigkeit leicht zu Worthülsen. Hayek prägte für die Begriffsproblematik der „sozialen Gerechtigkeit“ die Bezeichnung des „Wieselwortes“. Dies lässt sich auch auf den Begriff der Generationengerechtigkeit übertragen, der ob seiner möglichen Vieldeutigkeit letztlich inhaltsleer wird.

Eine Gefahr besteht nun darin, dass die im umgangssprachlichen Verständnis der Bevölkerung grundsätzlich positiv besetzten Gerechtigkeitsbegriffe in der politischen Praxis für die Durchsetzung von Partikularinteressen leicht instrumentalisiert werden können. Denn für die (praktische) Politik spielt die Art, wie bestimmte Themen und Sachverhalte in der breiten Bevölkerung wahrgenommen werden, durchaus eine wichtige Rolle. So können die in den regelmäßigen Umfragen vermittelten Stimmungen einen Handlungsdruck erzeugen und den politischen Entscheidungsprozess beeinflussen. In einem anderem Kontext stellt Sen zur Bedeutung der Wahrnehmung fest: „A perceived sense of inequity is a common ingredient of rebellion in societies. " 12 
Interessengruppen können also durch bewusst eingesetzte und selektive Informationen im Zusammenspiel mit den Medien einen Handlungsdruck erzeugen und auf politische Entscheidungen einwirken. Dies geschieht etwa auch dadurch, dass mit positiven Assoziationen verbundene Begriffe wie Gerechtigkeit herausgestellt werden und mit entsprechendem Zahlenmaterial unterlegt ein Bild gezeichnet wird, das in der Öffentlichkeit ein naives Unrechtsbewusstsein oder auch Ängste hervorruft bzw. Auffassungen unterstützt wie „gerecht ist, was gleich ist“. Dass Ungleichheit nicht zwangsläufig ungerecht sein muss, wurde bereits in Abschnitt II. dargestellt. Schroeder stellt hierzu fest: „Weil sich philosophische Konzepte nicht einfach in Politik umbrechen lassen, gehen Parteien und Regierungen von dem aus, was die Menschen als ungerecht empfinden.“13

Für die Meinungsbildung der Bevölkerung spielen die Medien eine erhebliche Rolle. Beispiele dafür sind in jüngster Zeit die Themen Managergehälter und Armut. Die Art der Berichterstattung ist ein Grund für die von Schroeder in der deutschen Bevölkerung festzustellende Fehlwahrnehmung bei der Einschätzung der Einkommens-, Vermögens- und Armutsverteilung bzw. -position. ${ }^{14}$ Die eigene Position wird von der Mehrheit systematisch unterschätzt, was mit der Frequenz der Berichte über Millionengehälter erklärt werden kann. Andererseits ist für diese Einschätzung bemerkenswert, dass die Gehälter von Fußballprofis, Schauspielern oder Popstars deutlich weniger kritisch gesehen werden als jene von Managern.

Ähnliche „Fehlwahrnehmungen“ bestimmen auch die aktuelle Armuts- und Rentendiskussion. Mit dem Argument, dass am wirtschaftlichen Aufschwung auch die Rentner partizipieren sollen, wurde der sog. Riesterfaktor ausgesetzt, der seinerzeit als zentrales Element der Reformen zur Sicherung der Nachhaltigkeit in der gesetzlichen Rentenversicherung - und damit als Beitrag zur intergenerativen Gerechtigkeit - eingeführt worden war. Der politische Handlungsdruck wurde auch durch die zeitgleich geführte Diskussion um eine drohende Altersarmut verstärkt. Die „Fehlwahrnehmung“ besteht dabei zum einen darin, dass der Aufschwung auch bei der breiten Masse der aktiv Versicherten nicht zu spürbar höheren Einkommen geführt hat, was im System der GRV die notwendige Bedingung für Rentenerhöhungen darstellt. Zum anderen sind nach derzeitigem Kenntnisstand und Datenlage nicht die heutigen Rentner von Altersarmut be-

13 Schroeder, K.: Zu Begriff und Bedeutung von Sozialer Gerechtigkeit, in: Stiftung Marktwirtschaft (Hg.): Was ist sozial? Dimensionen der Gerechtigkeit, Berlin, 2007, 10-16, hier 11.

14 Ebd. 
droht, sondern allenfalls die zukünftigen Rentner. Dies belegen die altersspezifischen Armutsquoten, die auf der Basis des sozioökonomischen Panels (SOEP) für die Jahre 1986 und 2006 berechnet wurden (vgl. Abbildung 1).

Abbildung 1: Altersspezifische Armutsrisikoquoten

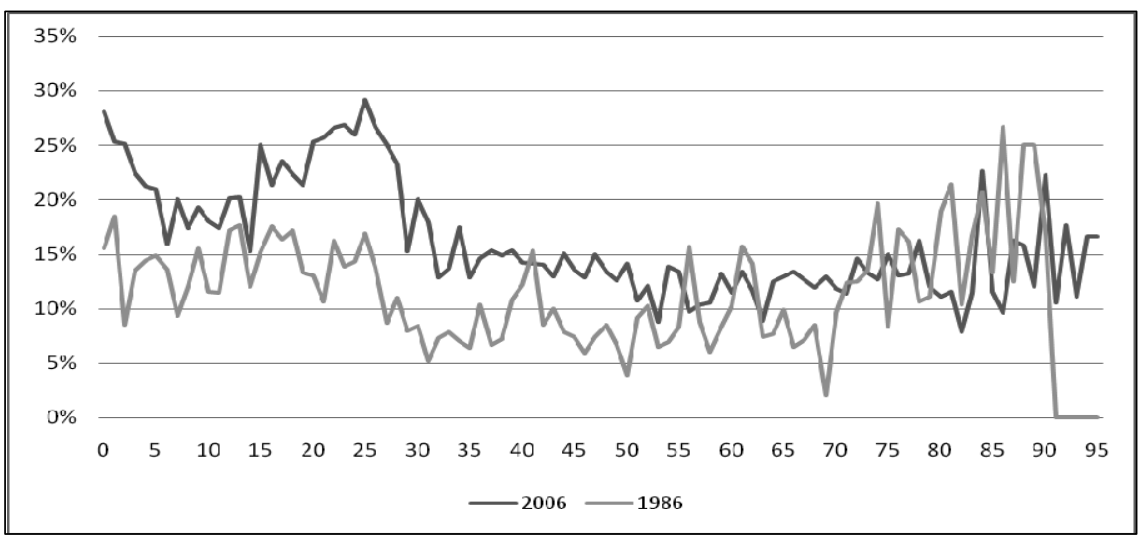

Quelle: SOEP, eigene Berechungen.

Aufgrund dieser Fakten kann ein dramatischer Anstieg der Altersarmut nicht festgestellt werden. Bedenklich sind eher die ansteigenden Armutsquoten in der Gruppe der unter 30-Jährigen. Für die Interpretation sind aber die methodischen Grundlagen der Bestimmung von Armutsquoten zu berücksichtigen, da sonst Fehlschlüsse nicht zu vermeiden sind. Anzumerken ist außerdem, dass Armut für die Beschreibung der aktuellen Situation in Deutschland ein eher ungeeigneter Begriff ist. Armut weckt Assoziationen mit den Zuständen in der dritten Welt und verunsichert die Bevölkerung mit der Gefahr der Instrumentalisierung. In Deutschland geht es aber nach den vorliegenden Daten nicht um ein Problem „absoluter" Armut. Denn selbst für Menschen mit geringen Einkommen gehören Waschmaschine, Fernseher und Computer heute zur Grundausstattung. Auch ein Vergleich der Ausgabenstruktur eines Sozialhilfe- bzw. ALG-II-Empfängers in den 1970er Jahren und heute zeigt, dass der Wohlstand in allen Bevölkerungsgruppen gestiegen ist. So gab ein Sozialhilfeempfänger früher etwa $50 \%$ des Budgets für Ernährung aus, heute sind dies beim ALG-II-Empfänger nur ca. $30 \%$. Für die Teilnahme am kulturellen Leben und die Befriedigung sonstiger persönlicher Bedürfnisse standen früher etwa $20 \%$ des Budgets zur Verfügung, 
heute sind es $40 \%$. In der aktuellen Diskussion geht es also um eine „relative“ Armut, die jedoch treffender mit dem Begriff der Ungleichheit bezeichnet wird.

Methodisch von Bedeutung ist die Arithmetik der relativen Armutsschwelle. Diese liegt bei $60 \%$ des Medianeinkommens. Wer weniger verdient gilt als arm. Die Armutsschwelle in Euro-Beträgen hängt neben der Datengrundlage auch davon ab, ob das selbstgenutzte Wohneigentum als Einkommensbestandteil berücksichtigt wird oder nicht. Dies erklärt, warum die Armutsschwelle im zweiten Armuts- und Reichtumsbericht der Bundesregierung auf Basis der Einkommens- und Verbrauchsstichprobe EVS 2003 bei 938 Euro pro Monat lag, ${ }^{15}$ während sie im dritten Armuts- und Reichtumsbericht auf Basis von EU-SILC-Daten mit 781 Euro pro Monat angegeben ist. ${ }^{16}$ Die Kenntnis dieser Sachverhalte und Zusammenhänge ist aber entscheidend dafür, dass die Bürger ihre eigene Situation realistisch einschätzen können.

Was die vergleichsweise hohen Armutsquoten der unter 30-Jährigen angeht, so ist dazu anzumerken, dass beispielsweise Studenten in der überwiegenden Mehrzahl immer als ,arm im Sinne der Statistik“ gezählt werden. Nach dem capabilities-Ansatz von Sen ist jedoch nur in Ausnahmefällen eine tatsächliche Armut gegeben bzw. wird von den Studierenden auch als solche empfunden. Schroeder unterscheidet in diesem Zusammenhang realitätsbezogen zwischen vorübergehender (temporärer) und dauerhafter Armut. ${ }^{17}$ Sozialpolitik ist zwar in allen Fällen gefordert, muss sich aber vor allem um die dauerhafte Armut und ihre Ursachen kümmern.

\section{Das Fallbeispiel „Soziale Pflegeversicherung“}

Die Soziale Pflegeversicherung (SPV) bietet ein weiteres Anschauungsbeispiel für die skizzierten Zusammenhänge von öffentlichem Gerechtigkeitsempfinden und politischen Entscheidungen. Dies wird bereits an den Entwicklungen im Zusammenhang mit ihrer Einführung deutlich:

Wie eingangs erwähnt, unterliegt die Abwägung von Solidarität und Subsidiarität dem jeweiligen Zeitgeist. Mit Einführung der SPV im Jahr 1995 hat die Politik eine Neubewertung der bis dato gültigen Balance zwischen Solidarität und Sub-

15 Bundesministerium für Arbeit und Soziales: Lebenslagen in Deutschland. Der zweite Armuts- und Reichtumsbericht der Bundesregierung, Berlin, 2005.

16 Bundesministerium für Arbeit und Soziales: Lebenslagen in Deutschland. Der dritte Armuts- und Reichtumsbericht der Bundesregierung, Berlin, 2008.

17 Schroeder, K., a. a. O. 
sidiarität vorgenommen. So wurde mit der Etablierung des fünften Sozialversicherungszweigs und das gleichzeitige Zurückdrängen der bedürftigkeitsgeprüften Sozialhilfe die Bedeutung des Subsidiaritätsprinzips reduziert. Die politische Rechtfertigung erfolgte mit dem Verweis auf eine vermeintliche Stärkung der Solidarität und damit dem Beitrag zur intragenerativen bzw. sozialen Gerechtigkeit. Die tatsächlichen Verteilungswirkungen stehen hierzu jedoch in krassem Widerspruch. Denn wirklich Bedürftige bekamen auch schon vor 1995 Leistungen, mehr schlecht als recht finanziert aus der kommunalen Sozialhilfe als „Hilfe zur Pflege“ ${ }^{18}$ Jedoch oblag es ihnen, ihre Mittellosigkeit nachzuweisen. Das ist nun anders - jeder ist leistungsberechtigt, egal ob bedürftig oder nicht.

Wer hat also gewonnen? Genau diejenigen, die nicht bedürftig sind. Den Tatbestand, dass sich an der Situation für Bedürftige nicht viel geändert hat, zeigt die Gegenüberstellung der Leistungsprofile der „Hilfe zur Pflege“ (HzP) sowie der SPV (Abbildung 2). Während rein aus Gründen der besseren Vergleichbarkeit das Leistungsprofil der HzP in Preisen des Jahres 2006 ausgedrückt ist, illustriert das so indexierte Leistungsprofil der HzP, dass dieses in seinem absoluten Niveau nahezu an das Leistungsprofil der SPV heranreicht. Demzufolge hat sich an der Situation für die ärmeren Teile der Bevölkerung nichts geändert - die Leistungen im Pflegefall wurden mit Einführung der SPV nun lediglich durch einen anderen Parafiskus erbracht. Demgegenüber änderte sich aber die Situation für die Nichtbedürftigen der Gesellschaft deutlich. Mit einem Schlag wurden nun auch Ihnen Leistungen zuteil, auf die sie aufgrund ihrer Einkommens- und/oder Vermögenssituation zuvor keinen Anspruch hatten. Hierin einen Beitrag zur intragenerativen Gerechtigkeit zu sehen, bedarf einiger Phantasie, zumindest widerspricht es dem landläufigen Gerechtigkeitsempfinden, das mehr Gleichheit für gerechter hält. Und mehr noch, die von der Politik als Beitrag zur intragenerativen bzw. sozialen Gerechtigkeit proklamierte Maßnahme ging voll zu Lasten künftiger Generationen bzw. wurde auf Kosten einer intergenerativen Ungleichheit erkauft, die nur schwer mit einem Gerechtigkeitskonzept vereinbar scheint.

18 Tatsächlich lag ein wesentlicher Grund für die Einführung der SPV in den prekären Kommunalhaushalten und der Schwierigkeit bei der Einigung auf einen Finanzausgleich der Länder. 
Abbildung 2: Altersspezifische Leistungsprofile der „Hilfe zur Pflege“ und der SPV

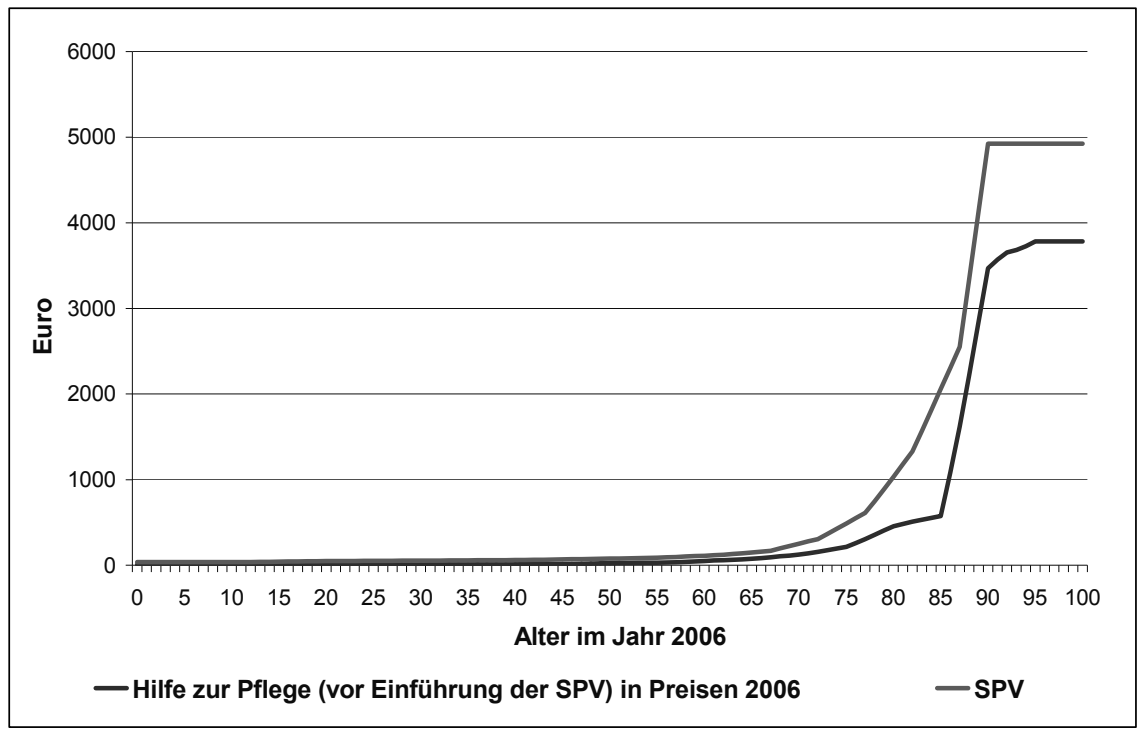

Quelle: Eigene Darstellung.

So sind alle heutigen Pflegefälle statistisch gesehen die „Einführungsgewinnler“ eines neu begründeten Kettenbriefs. Im Gegensatz zu einem kapitalgedeckten System, in dem zur Leistungsgewährung erst eine vorgelagerte Phase der Kapitalakkumulation notwendig ist, werden im Rahmen des Umlageprinzips die laufenden Beitragseinnahmen zur Finanzierung der in der gleichen Periode anfallenden Leistungsausgaben verwendet. Damit ist die Versorgung der Bestandsfälle und der risikonahen Fälle zum Zeitpunkt der Systemimplementierung sichergestellt. Demzufolge beschert also das Umlageverfahren all jenen Generationen einen „Gewinn“ bzw. ein „Geschenk“, die zum Zeitpunkt der Einführung und in den darauffolgenden Jahrzehnten zum Kreis der Leistungsempfänger gehören, ohne vorher entsprechende Beiträge geleistet zu haben.

Dieser Vorteil der ersten Generationen stellt sich aber nur bei entsprechender demographischer Entwicklung bzw. Wirtschaftswachstum und Beschäftigungslage als tatsächlicher Vorzug eines Umlageverfahrens heraus. Denn nur wenn die Rendite aus dem umlagebasierten System größer ist als jene aus einem alternativen kapitalgedeckten System, stellt die Einführung eines Umlageverfahrens für 
alle Generationen einen Vorteil dar. Im umgekehrten Fall ruft jede Inkraftsetzung des Umlageprinzips sowohl Gewinner als auch Verlierer hervor, wobei Letztere umso stärker als „Verlierer“ hervorgehen, je mehr die Bevölkerung eine Alterungstendenz aufweist. ${ }^{19}$ Da das Pflegerisiko ein fast reines Altersrisiko ist, folgt, dass im Zuge des demographischen Alterungsprozesses, also mit steigendem Anteil älterer und damit pflegebedürftiger Menschen an der Gesamtbevölkerung und mit abnehmendem Anteil erwerbstätiger Beitragszahler, die beitragspflichtigen Versicherten entsprechend stärker zur Finanzierung der Leistungen herangezogen werden müssen. Dies hat laufende Beitragssatzanhebungen zur Folge.

Abbildung 3: Altersspezifische Beitrags- und Leistungsprofile der SPV

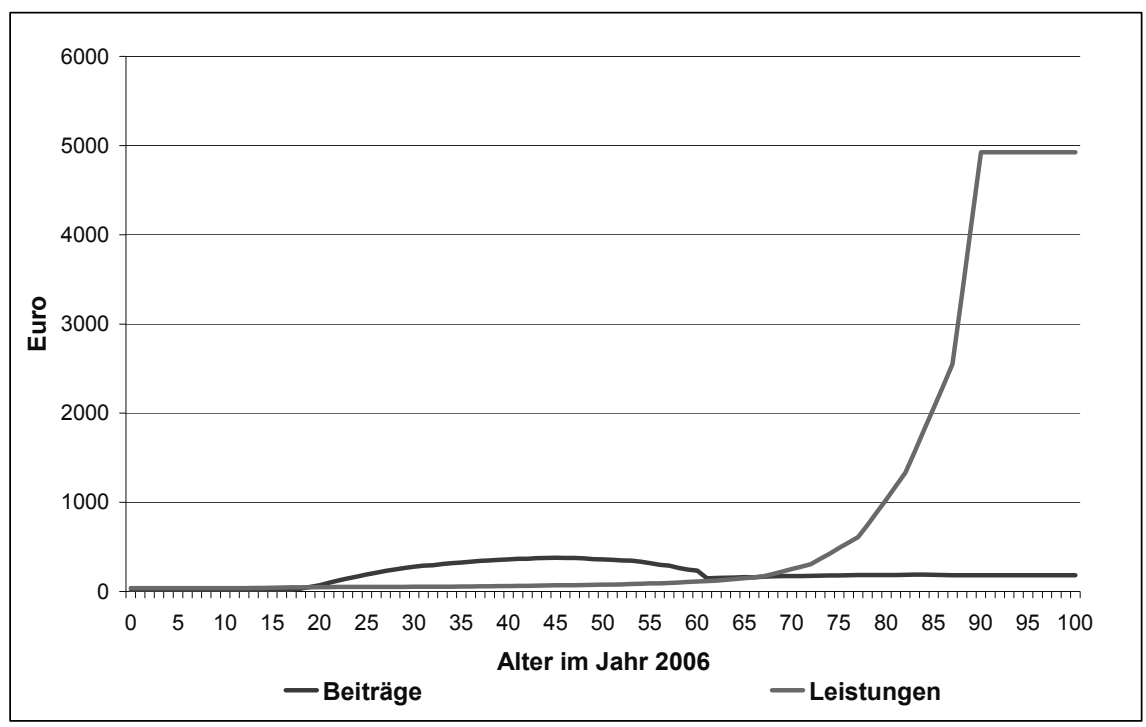

Quelle: Eigene Darstellung.

Was also wurde aus intergenerativer Sicht mit Einführung der SPV auf Umlagebasis eigentlich erreicht? Ein Blick auf Abbildung 3 zeigt, dass die durchschnittlichen Leistungsausgaben der SPV pro Kopf und Jahr bis zu einem Alter von

19 Für eine umfassende Analyse zu internen Renditen in der SPV siehe Häcker, J./Raffelhüschen, B.: Die Interne Rendite der Gesetzlichen Pflegeversicherung, in: Zeitschrift für die gesamte Versicherungswissenschaft, 95/2 (2006), 267-286. 
etwa 65 Jahren verschwindend gering sind, dann aber exponentiell ansteigen. Demgegenüber erscheinen die durchschnittlichen Beitragszahlungen, die von den entsprechenden altersspezifischen Lohnprofilen abhängen, verschwindend gering. Der Generationenvertrag SPV ist leicht zu identifizieren, denn zwischen 20 und 65 zahlt der Durchschnittsversicherte deutlich mehr in die Pflegeversicherung ein, als er von ihr erhält. Ist er/sie schließlich älter als 65 , dann sind die Ansprüche deutlich höher als die Einzahlungen. Und gemäß dem Recht des Generationenvertrages ist dies deshalb gerecht, weil jeder gegenwärtig Alte irgendwann einmal jung gewesen sein muss und entsprechend eine Phase gehabt hat, in der er mehr eingezahlt hat, als er im Gegenzug von der Pflegeversicherung erhielt.

Ein gleich zweifaches Unbehagen sollte den aufmerksamen Leser an dieser Stelle ergreifen: Erstens kann es dieses Recht des Generationenvertrages nur dann geben, wenn er schon hinreichend lange bestand, und zweitens stimmt schon der schiere Flächenvergleich zwischen Ein- und Auszahlungen des „Generationenvertrags SPV“ nachdenklich. Um es auf den Punkt zu bringen: Die heutigen Pflegebedürftigen können durch vergangene Einzahlungsströme gar kein Recht auf Leistung erworben haben, weil es die SPV so lange noch nicht gibt. Ein echter Bestandsschutz - wie in der Renten- oder Krankenversicherung - existiert also noch nicht. Wenn überhaupt, genießt dieser Personenkreis Vertrauensschutz, weil er auf ein falsches politisches Versprechen baute. Dieses Versprechen ist jedoch für die lange Frist und damit für zukünftige Generationen von Pflegbedürftigen nicht einzuhalten. Schlimmer noch: Aus diesen altersspezifischen Beitrags- und Leistungsprofilen kann man ableiten, dass das, was eigentlich ein Generationenvertrag sein müsste, heute schon keiner ist und genau genommen niemals einer war.

Addiert man für jeden durchschnittlichen Beitragszahler aus Abbildung 3 den Barwert der Einzahlungen über den restlichen Lebenszyklus und zieht hiervon den Barwert der statistisch erwarteten Leistungsansprüche ab, so ergibt sich sein entsprechendes Generationenkonto. Aus den Generationenkonten aller lebenden Durchschnittsmenschen leitet man die Generationenbilanz der SPV ab - und genau diese Bilanz zeichnet ein erschreckendes Bild (vgl. Abbildung 4): ${ }^{20}$ Alle heute lebenden Jahrgänge weisen negative Generationenkonten auf, werden also unter dem gegenwärtigen gesetzlichen status quo mehr Leistungen erhalten als

20 Vgl. für alle Detailinformationen Häcker, J.: Die Soziale Pflegeversicherung: Eine Generationenbilanz, Frankfurt a. M., 2008. 
Beiträge zahlen. Damit war bzw. ist die SPV noch nie ein Generationenvertrag gewesen, eben weil es nie auch nur einen lebenden Jahrgang gegeben hat bzw. geben wird, der mit einem Beitrag von 1,95\% den statistischen Erwartungswert seiner Leistungsinanspruchnahme auch einzahlt. ${ }^{21}$ Nicht junge Erwerbstätige zahlen an alte Pflegebedürftige, sondern alle heute lebenden Generationen bereichern sich zu Lasten ihrer Kinder und Kindeskinder.

\section{Abbildung 4: Generationenbilanz der SPV im status quo}

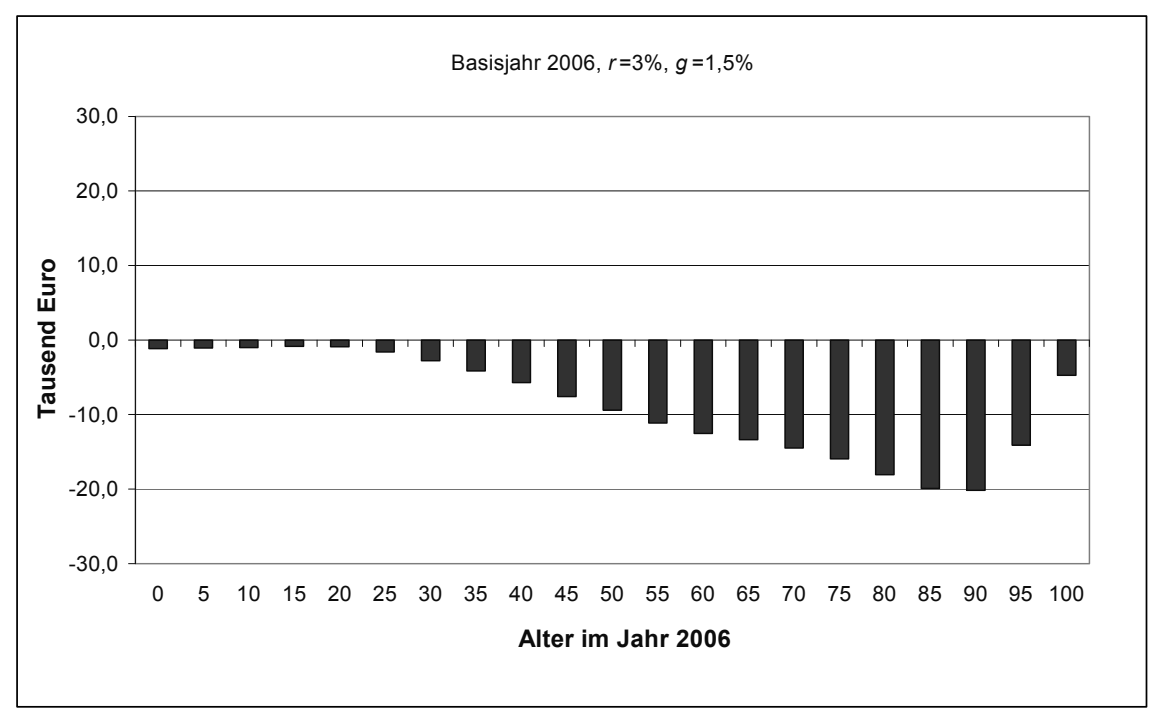

Quelle: Eigene Darstellung

21 Der Beitragssatz von 1,95 \% ist das Ergebnis des zum 01.07. 2008 in Kraft getretenen Pflege-Weiterentwicklungsgesetzes (PflWG). Für die Zeit vom 01.01.1995 bis zum 30.06.1996 betrug der Beitragssatz bundeseinheitlich $1 \%$, mit Gewährung der stationären Pflegeleistungen seit dem 01.07.1996 beträgt er $1,7 \%$ des beitragspflichtigen Einkommens. Darüber hinaus gilt seit dem 01.01.2005, dass kinderlose Versicherte einen Aufschlag von 0,25 Prozentpunkten leisten müssen. Dieser sogenannte Familienlastenausgleich ist die Konsequenz eines am 03.04. 2001 verkündeten Urteils des Bundesverfassungsgerichts (BVerfGE 103, $242 \mathrm{ff}$.). Hierin stellte das Bundesverfassungsgericht fest, dass es nicht verfassungskonform sei, ,[...] dass Mitglieder der sozialen Pflegeversicherung, die Kinder betreuen und erziehen [und damit neben dem Geldbetrag auch einen generativen Beitrag zur Funktionsfähigkeit eines umlagefinanzierten Sozialversicherungssystems leisten], mit einem gleich hohen Pflegeversicherungsbeitrag wie Mitglieder ohne Kinder belastet werden“. Letztendlich wurde dieses Urteil von der damaligen Gesundheits- und Sozialministerin Ulla Schmidt nicht durch eine Entlastung der Versicherungsmitglieder mit Kindern umgesetzt, sondern vielmehr durch eine Belastung von Mitgliedern ohne Kinder. 
Welche Lasten genau auf diese zukünftigen Generationen zukommen, zeigt die aus der Generationenbilanz abgeleitete Nachhaltigkeitslücke. Diese entspricht der mit der Jahrgangsstärke gewichteten Summe aller Generationenkonten aller lebenden und zukünftigen Generationen. Vereinfacht ausgedrückt reflektiert die Nachhaltigkeitslücke den Betrag, der fehlt, um das derzeitige Leistungsniveau bei gegebenen Beiträgen zur SPV auch für zukünftige Generationen aufrechterhalten zu können. Diese quasi unverbriefte und deshalb nirgendwo statistisch erfasste Verschuldung der SPV beträgt selbst unter der unrealistisch optimistischen Annahme, dass im Pflegebereich zukünftig kein Kostendruck auftritt, etwa ein Drittel des Bruttoinlandsprodukts. Dies sind etwa 0,7 Billionen Euro. In einem etwas realistischeren Szenario eines aber immer noch optimistisch geringen Kostendrucks liegt die Nachhaltigkeitslücke bereits bei knapp $55 \%$ dessen, was man in Deutschland in einem Jahr an Gütern und Dienstleistungen produziert. Wie man es auch dreht und wendet, eines liegt auf der Hand: Die Einführung der SPV hat die Staatsverschuldung in Deutschland mit einem Federstrich mindestens verdoppelt. Gesehen hat dies aber kaum jemand, weil unverbriefte Schulden der Generationenverträge eben statistisch nicht in den Haushaltsrechnungen erfasst werden.

Angesichts dieses Sachverhalts ist es nicht verwunderlich, dass die SPV in einer alternden Gesellschaft in massive Finanzierungsschwierigkeiten gerät. Will man das gegenwärtige reale Leistungsniveau unter realistischen demographischen Annahmen sichern, so erfordert dies eine Steigerung des Beitragssatzes auf über $3 \%$ im Jahr 2040 und auf 4,3\% im Jahr 2055. Dies reflektiert aber lediglich eine Untergrenze - unter Berücksichtigung eines zweifellos auftretenden Kostendrucks dürften die entsprechenden Werte eher bei über $4 \%$ im Jahr 2040 und knapp $6 \%$ im Jahr 2055 liegen. $^{22}$

Das Versprechen auch für die heutigen Erwerbstätigen eine Absicherung der Pflegebedürftigkeit zu gewährleisten, ist also offensichtlich nicht einzuhalten. Denn wenn diese geburtenstarken Jahrgänge das entsprechende Alter erreichen, haben die zukünftigen Beitragszahler keine andere Wahl, als den „Generationenvertrag, der nie einer war" aufzukündigen. Die Einführung der SPV war ein historischer Etikettenschwindel; ehrlicherweise hätte man die ursprüngliche Gesetzesinitiative eher „Gemeindefinanzreformaufschubsgesetz“ oder „Erb-

22 Vgl. hierzu auch Häcker, J., a. a. O. Quantitative Unterschiede ergeben sich aufgrund der Wahl eines neuen Basisjahres und aufgrund der Berücksichtigung der 11. koordinierten Bevölkerungsvorausberechnung des Statistischen Bundeasamts. 
schaftsbewahrungsgesetz" nennen sollen. Letzteres deshalb, weil vor der Einführung einzig und allein Bedürftige und nicht vermögende Pflegefälle durch die Sozialhilfe abgesichert waren.

Der mit Einführung der SPV entstandene Verschiebebahnhof zwischen intraund intergenerativer Umverteilung wurde ganz aktuell mit dem Pflege-Weiterentwicklungsgesetz (PflWG) erneut strapaziert. Maßgeblich dazu beigetragen hat der in $§ 14$ Abs. 4 SGB XI gefasste Begriff der Pflegebedürftigkeit und der entsprechend festgesetzte Hilfebedarf. So begrenzt sich der bei Feststellung einer Pflegebedürftigkeit berücksichtigungsfähige Hilfebedarf auf Verrichtungen im Ablauf des täglichen Lebens wie Körperpflege, Ernährung, Mobilität und Hauswirtschaft. Nicht berücksichtigt ist hingegen der darüber hinausgehende Beaufsichtigungs- und Betreuungsaufwand für die Pflege des in der öffentlichen Diskussion vereinfachend als demenzkrank bezeichneten Personenkreises. Im Rahmen des PflWG wird dieser Unzulänglichkeit nun entgegengewirkt und eine Gleichbehandlung demenziell erkrankter Personen erwirkt. ${ }^{23}$ Jedoch wird die stärkere Gleichbehandlung auf intragenerativer Ebene wiederum auf Kosten einer stärkeren Ungleichbehandlung auf intergenerativer Ebene, also zu Lasten zukünftiger Generationen, erkauft. Denn durch die Gleichstellungspolitik des PflWG wurde eine neue Runde von Einführungsgeschenken verteilt - die zudem an einen Personenkreis gehen, der ohnehin von der Einführung der SPV als Umlageverfahren profitiert hat - und damit eine weitere implizite Schuld auf das System der SPV geladen.

Gehört es aber nicht zu einem umfassenden Gerechtigkeitsbegriff, intra- und intergenerative Aspekte in eine Balance zu bringen - ohne, dass dies zwingend eine exakte Gleichverteilung der fiskalischen Lasten bedeutet? Bezogen auf das PflWG stellt sich die Frage, wie die bestehenden Ansprüche zu bedienen sind, und damit auch, wie gesichert werden kann, dass die Personen, die seit Inkrafttreten des SGB XI die aktuellen Pflegejahrgänge finanzieren, diese Solidarität später auch für sich bei alternder Bevölkerung erwarten können.

Abschließend ist noch auf eine weitere Kuriosität der vermeintlich am Gerechtigkeitsideal orientierten SPV einzugehen. Rein fiskalisch betrachtet tritt neben der intergenerativen und sozialen Ungleichheit außerdem eine Geschlechterungleichheit innerhalb des Systems der SPV hervor, die in den geschlechtsspezi-

23 Das Bundesgesundheitsministerium hat zudem im November 2006 einen Beirat eingerichtet, der die bestehende Regelung kritisch überprüfen soll und von dem für Ende des Jahres 2008 konkrete Änderungsvorschläge zu erwarten sind. 
fischen durchschnittlichen Beiträgen und Leistungen zum Ausdruck kommt (Abbildung 5): So leisten Frauen im Vergleich zu den Männern nur etwa die Hälfte der Beitragszahlungen, erhalten gleichzeitig aber deutlich mehr Pflegeleistungen, was auf die längere Lebenserwartung und der im Durchschnitt damit verbundenen umfangreicheren Transfererhalte der Frauen zurückzuführen ist. Die geringeren durchschnittlichen Beitragszahlungen der Frauen sind dabei das Resultat der im Vergleich zu den Männern geringeren Erwerbsbeteiligung sowie der niedrigeren Durchschnittseinkommen.

Abbildung 5: Alters- und geschlechtsspezifische Beitrags- und Leistungsprofile der SPV

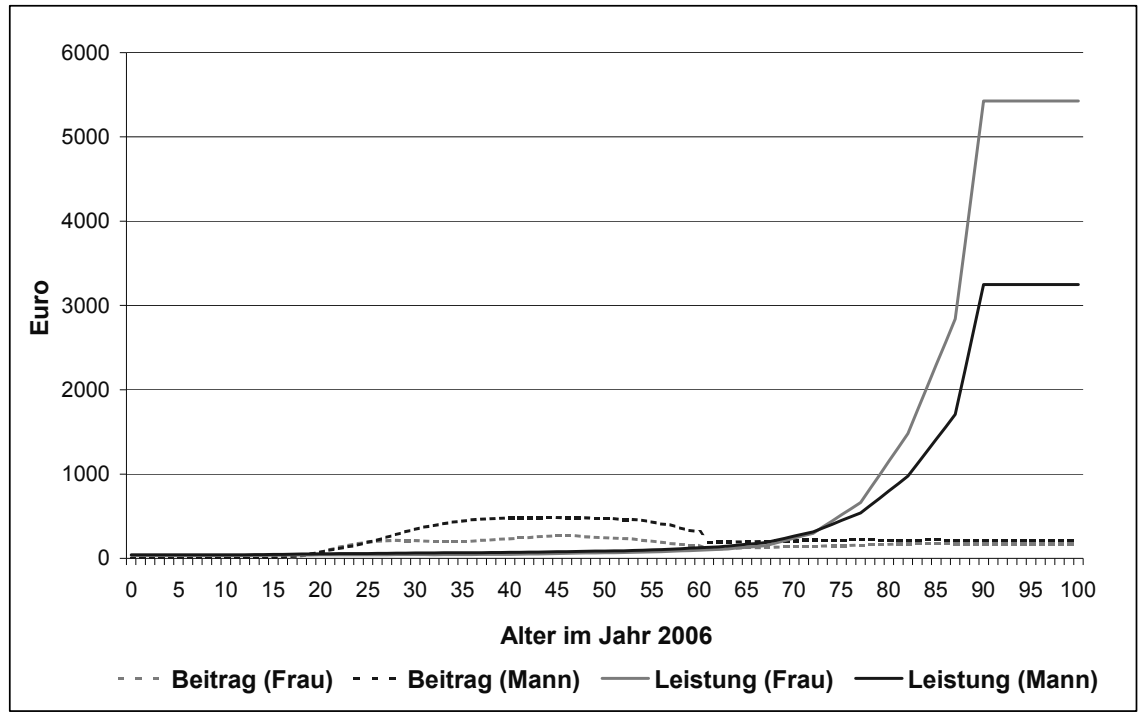

Quelle: Eigene Darstellung.

Ein Vergleich zu den risikoäquivalenten Prämienzahlung, wie sie im Rahmen einer kapitalgedeckten Pflegeversicherung bei gleichen Leistungstransfers fällig werden würde, zeigt, dass diese für Frauen stets über den Beitragszahlungen zur SPV liegt. So müssen die Frauen in der kapitalgedeckten Variante exakt den „Preis“ für Pflegeleistungen zahlen, der ihrem Risikoprofil entspricht. Dagegen würden Männer im Rahmen der kapitalgedeckten Variante tendenziell entlastet, da sie zwar ebenfalls eine risikoäquivalente Prämie leisten müssten, diese aber deutlich unter ihren Beitragszahlung zur SPV liegen würde. Dieser Vergleich 
offenbart damit auch die im Rahmen der SPV stattfindende intragenerative Umverteilung zwischen Männern und Frauen. So findet im jetzigen System der SPV eine erhebliche intragenerative Umverteilung zu Gunsten der Frauen statt, was angesichts der gesellschaftlich anerkannten Gleichheit von Mann und Frau erstaunen kann. ${ }^{24}$

Aber ist diese fiskalisch dokumentierte Ungleichheit auch ungerecht? Schließlich lässt die Analyse von reinen Euro-Beträgen außer Acht, dass Frauen familiale bzw. unentgeltliche Pflegeleistungen erbringen, ohne welche die SPV deutlich höhere Ausgaben zu verzeichnen hätte. Ohne die familiale Unterstützungsleistungen der Frauen müssten Pflegebedürftige in erhöhtem Maße professionelle Dienste in Anspruch nehmen, was eine vermehrte Substitution von Geld- zu Sachleistungen und von ambulanter zu stationärer Pflege zur Folge hätte. ${ }^{25}$ Denn Voraussetzung für die Inanspruchnahme von häuslicher Pflege ist gemäß den Pflegeberichten des Medizinischen Dienstes der Spitzenverbände der Krankenkassen das Vorhandensein einer familialen Pflegeperson. ${ }^{26}$ So werden laut der Infratest Sozialforschung $92 \%$ aller in Privathaushalten versorgten Pflegebedürftigen von Familienangehörigen betreut. Dabei sind rund $73 \%$ der familialen Pflegepersonen weiblichen Geschlechts. ${ }^{27}$ Ist damit die monetäre Besserstellung der Frauen nun doch gerecht? Das Beispiel verdeutlicht, dass im Zuge von Gerechtigkeitsdiskussionen immer viele Dimensionen ausschlaggebend sind und dass ,gerecht“ nicht immer ,gleich“ bedeutet. Erneut wird deutlich, dass die praktische Umsetzung des theoretischen Begriffs „Gerechtigkeit“ nicht trivial ist. Nicht nur, dass sich nicht alle Tatbestände quantifizieren lassen, auch führen unvollständige bzw. selektiv gebotene Informationen zu Fehlwahrnehmungen, die wiederum politischen Handlungsdruck erzeugen können.

\section{Fazit}

Gerechtigkeit ist ein Begriff, der häufig zur Begründung bzw. Rechtfertigung politischer Entscheidungen und Maßnahmen, aber auch zur Beschreibung sub-

24 Für eine detaillierte Analyse hierzu vgl. auch Häcker, J./Raffelhüschen, B., a. a. O.

$25 \mathrm{Zu}$ den fiskalischen Effekten resultierend aus der Substitution von familialen durch professionelle Pflegeleistungen siehe Häcker, J./Raffelhüschen, B.: Zukünftige Pflege ohne Familie: Konsequenzen des „Heimsog-Effekts“, in: Zeitschrift für Sozialreform, 53/4 (2007), 391-422.

26 Medizinischer Dienst der Spitzenverbände der Krankenkassen e.V.: Pflegebericht des Medizinischen Dienstes, Essen, 1998; ders.: Pflegebericht des Medizinischen Dienstes, Essen, 2000.

27 Infratest Sozialforschung: Hilfe- und Pflegebedürftige in Privathaushalten in Deutschland 2002. Schnellbericht, München, 2003. 
jektiver Befindlichkeiten und individueller Einschätzungen herangezogen wird. Eine differenzierte Betrachtung zeigt jedoch, dass mit dem Begriff sowohl im umgangssprachlichen wie politischen Gebrauch teilweise ganz unterschiedliche Aspekte beschrieben und Dimensionen angesprochen werden, die teilweise in Konflikt zueinander stehen und damit ein sachadäquate Diskussion erschweren bzw. unmöglich machen. Eine für den praktischen politischen Prozess notwendige Gewichtung der jeweils angesprochenen Dimensionen und damit eine Operationalisierung des Begriffs ist wissenschaftlich kaum zu begründen. Weil zudem jeder etwas anderes unter diesem Begriff versteht oder verstehen kann, ist der Gerechtigkeitsbegriff für die praktische Politik äußerst problematisch. Hinzu kommt, dass der Begriff umgangssprachlich positiv besetzt ist und deshalb zur bewusst missbräuchlichen Verwendung verleitet. Die aktuellen politischen Diskussionen zeigen deutlich, dass Parteien und Interessengruppen mit Verweis auf die (soziale) Gerechtigkeit Emotionalisierung und Handlungsdruck erzeugen, um ihre Partikularinteressen durchzusetzen.

Die dargestellten Beispiele der Armuts- und Rentendiskussion sowie der SPV zeigen aber, dass in der Öffentlichkeit durch Unkenntnis verursachte erhebliche Fehlwahrnehmungen zu diesen Sachverhalten und zu (Verteilungs-)Wirkungen bestimmter Maßnahmen bestehen. Darüber hinaus wird offensichtlich, dass Maßnahmen, die der Öffentlichkeit von Seiten der Interessengruppen bzw. der Politik als der Gerechtigkeit dienlich vermittelt werden, bestenfalls eine Dimension der Gerechtigkeit betreffen. So ist die jüngste Rentenanpassung mit dem Ziel, die Rentner am Aufschwung partizipieren zu lassen, zunächst durchaus nachvollziehbar. Bedenklich ist aber, dass zu diesem Zweck eine langfristige Strategie zur Austarierung der Interessen von aktiv Versicherten und Rentnern leichtfertig ausgesetzt wurde - die Vermutung liegt nahe, dass aus der Aussetzung auch eine Abschaffung werden kann. Der Glaubwürdigkeit der Politik wurde damit ein Bärendienst erwiesen. Dies gilt auch für die SPV, mit der den ärmeren Bevölkerungsteilen geholfen werden sollte, mit der aber effektiv das Gegenteil erreicht wird, weil Leistungen, die bisher nur Bedürftigen zustanden, nun allen zustehen. Wie dies ein Beitrag zur intragenerativen Gerechtigkeit sein soll, ist kaum zu begründen. Und auch als Beitrag zur intergenerativen Gerechtigkeit kann dies, aufgrund der dargestellten Sachverhalte kaum gesehen werden.

Was auch immer man politisch für intra- oder intergenerativ gerecht oder ungerecht hält, vom wissenschaftlichen Standpunkt bleibt dies eine nicht lösbare Werturteilsentscheidung. Und auch in diesem Kontext angewandte Methodik der Generationenbilanzierung ist grundsätzlich kein normatives Maß für Generatio- 
nengerechtigkeit. Sie postuliert daher auch nicht, dass die berechnete Nachhaltigkeitslücke in praxi vollständig geschlossen werden muss, denn schließlich ist unklar, ob kommende Generationen über eine größere oder kleinere Ressourcenausstattung verfügen werden - zumindest wenn ökologische, bildungspolitische oder noch breitere gesellschaftspolitische und historische Aspekte in die Beurteilung mit einbezogen werden, wie dies am abschließenden Beispiel des Geschlechterunterschieds in der SPV deutlich wurde. Und selbst wenn wir alle intergenerativen Verteilungsdimensionen quantitativ erfassen könnten, bleibt es immer noch Aufgabe des politischen Abstimmungs- und Entscheidungsprozesses, über eine ,gerechte“ Verteilung zu bestimmen.

Hier liegt dann auch der eigentliche Nutzen der Generationenbilanzierung. Indem sie Transparenz hinsichtlich der fiskalischen Ungleichbehandlung der Generationen schafft, erschwert sie eine für die Politik attraktive Verschleierungstaktik der Umverteilung zu Lasten künftiger Generationen. Um die Interessen der Generationen auszutarieren sollte die Politik deshalb dieses Instrument nutzen, um Partikularinteressen zu entlarven und so eine verantwortungsbewusste Haushaltspolitik in der Öffentlichkeit besser zu vermitteln. Abschließend ist mit Verweis auf das Eingangszitat von Kelsen festzustellen, dass die Generationenbilanzierung einen Beitrag dazu leistet, bessere Fragen zu stellen und so Zukunftsprobleme besser fassen und lösen zu können. 